\title{
The role of regulated protein degradation in auxin response
}

\author{
Sunethra Dharmasiri and Mark Estelle \\ Institute for Cellular and Molecular Biology, Section of Molecular Cell and Developmental Biology, University of \\ Texas at Austin, Austin, TX 7871, USA
}

Received 1 June 2001; accepted is revised form 9 July 2001

Key words: auxin, cullin, Nedd8, RUB, SCF, ubiquitin

\begin{abstract}
Auxin-regulated gene expression is mediated by two families of transcription factors. The ARF proteins bind to a conserved DNA sequence called the AuxRE and activate transcription. The Aux/IAA proteins repress ARF function, presumably by forming dimers with ARF proteins. Recent genetic studies in Arabidopsis indicate that auxin regulates this system by promoting the ubiquitin-mediated degradation of the Aux/IAA proteins, thus permitting ARF function. Mutations in components of $\mathrm{SCF}^{\mathrm{TIR} 1}$, a ubiquitin protein ligase (E3) result in stabilization of Aux/IAA proteins and decreased auxin response. Further, recent biochemical experiments indicate that the Aux/IAA proteins bind $\mathrm{SCF}^{\mathrm{TIR} 1}$ in an auxin-dependent manner.
\end{abstract}

\section{Introduction}

Auxin plays a crucial role in diverse aspects of plant growth and development including tropic responses, apical dominance in the shoot, lateral root formation and differentiation of the vascular system. To understand the molecular basis of auxin action, two major approaches have been used. The first approach is to identify mutants that lack normal auxin responses, and to determine the genetic basis for these defects (Hobbie et al., 1994). The second is to take a direct molecular approach to identify genes and proteins that are regulated by the auxin signal (Abel and Theologis, 1994; Guilfoyle et al., 1998). Both of these approaches have provided significant insights into auxin signaling. One of the most exciting developments of the past few years, at least from our perspective, is the convergence of the two approaches. In this review we will focus on recent results that indicate auxin response depends on the regulated degradation of a large family of transcriptional regulators, the Aux/IAA proteins.

\section{Ubiquitin proteasome pathway}

Ubiquitin is a small conserved protein that is covalently attached to diverse proteins, usually targeting them for degradation by $26 \mathrm{~S}$ proteasome (Hershko and Ciechanover, 1998). The involvement of regulated protein degradation by the ubiquitin-proteasome pathway has been demonstrated in a wide variety of cellular processes (Hershko and Ciechanover, 1998). The ubiquitin conjugation pathway begins by the ATP-dependent activation of ubiquitin by a ubiquitinactivating (E1) enzyme. All E1 enzymes have a conserved cysteine residue that forms a thiol ester linkage with the C-terminal glycine of ubiquitin. Usually, E1 enzymes are encoded by a single gene or a small family of related genes (Hershko and Ciechanover, 1998). The activated ubiquitin molecule is then transferred onto the second component of the pathway, a ubiquitin-conjugating (E2) enzyme, again forming a thiol ester linkage between the terminal glycine of ubiquitin and a conserved cysteine on the E2 enzyme. The E2 enzymes are encoded by a large gene family. In yeast, there are 11 related E2 enzymes while in Arabidopsis there are at least 36 Arabidopsis $\mathrm{E} 2$ isoforms belonging to 12 groups (Hershko and Ciechanover, 1998; R. Vierstra, personal communication). The third step in the ubiquitination reaction is the transfer of ubiquitin onto the substrate protein. This step is assisted by an E3 ubiquitin ligase that confers substrate specificity to the pathway. The ubiquitinated protein is 
then degraded by the $26 \mathrm{~S}$ proteasome, a large complex that comprises over 30 subunits, arranged in two subcomplexes of 19S and 20S (Hershko and Ciechanover, 1998; Vierstra and Callis, 1999). The 19S subcomplex is responsible for recognizing the tagged protein, while the lumen of the 20S subcomplex degrades the protein.

Compared to the E1 and E2 enzymes, E3 ligases are very diverse and complex. So far, four types of E3 ligases have been characterized. These are; HECT (homology to E6-AP carboxyl terminus) domain containing, Ubr1p (ubiquitin amino-end recognizing protein 1), APC/C (anaphase-promoting complex/cyclosome), and SCF (Skp1-cullin-F-box/RINGH2) E3 ligases. All four types have been identified in plants (Callis and Vierstra, 2000).

\section{SCF E3 ligases}

The SCF family of E3 ligases has been well characterized in plants, animals and yeast, both structurally and functionally (Patton et al., 1998). The name of the complex comes from the first three subunits identified in yeast, Skp1, Cdc53 (or cullin) and the F-box protein. Recently, a fourth subunit, a RING-H2 motif containing protein (Rbx1/Hrt1/Roc1) was recognized as an essential component of the complex (Tyers and Willems, 1999). The architecture of the SCF complex derived from protein interaction studies and structural analyses, suggests a common protein core consisting of Cullin, Rbx1 and Skp1. This core recruits different F-box proteins to form functionally distinct SCFs (Patton et al., 1998; Tyers and Willems, 1999). There is only a single Skp1 protein identified so far in man, but there are $18 \mathrm{Skp} 1$-related genes present in the Arabidopsis genome (called ASKs) (Crosby, personal communication). Cullins also belong to gene families. For example, there are at least six cullins in man and five in Arabidopsis. The F-box proteins function in substrate recognition and are the most diverse and specific components of the complex. Genome sequencing projects in Arabidopsis and Caenorhabditis elegans have identified over 300 F-box-containing proteins in each organism. Some of these F-box proteins may function in non-SCF complexes (Kipreos and Pagano, 2000; Galan et al., 2001). Nonetheless, the large number of F-box-containing protein encoding genes underscores the potential importance of SCF complexes and regulated protein degradation in various cellular processes.
Among the best-known SCF complexes in yeast is $\mathrm{SCF}^{C d c 4}$. This E3 facilitates degradation of Sic1 (an inhibitor of Cdc28/cyclinB), and promotes G1/S transition of the cell cycle. Yeast SCF ${ }^{G r r} 1$ promotes degradation of the G1 cyclins, Cln 1 and $\mathrm{Cln} 2$, and $\mathrm{SCF}^{\text {Met30 }}$ is involved in sulfur metabolism (Patton et al., 1998). In mammalian cells, $\mathrm{SCF}^{S k p 2}$ regulates the destruction of P27 ${ }^{k i p 1}$, an inhibitor of cyclindependent kinase Cdk2 (Carrano et al., 1999). In Arabidopsis, F-box proteins regulate jasmonic acid response (Coi1), floral development (UFO), circadian rhythm (ZTL and FKF1) (Xie et al., 1998; Samach et al., 1999; Nelson et al., 2000; Somers et al., 2000), and phytochrome-dependent light signaling (Dieterle et al., 2001). The SCF ${ }^{\mathrm{TIR} 1}$ complex is required for auxin response, and will be discussed in detail below (Gray et al., 1999).

\section{The ubiquitin-like proteins}

In addition to ubiquitin, several families of ubiquitin like proteins (Ubls) have been described (Vierstra and Callis, 1999; Hochstrasser, 2000; Yeh et al., 2000). As early as 1987, UCRP/ISG15, a ubiquitin-like protein modifier, was identified in animals followed by the SUMO/Sentrin family and the NEDD8/RUB family (Yeh et al., 2000). Ubls are activated and conjugated to the substrate in a mechanism similar to that of ubiquitin. However, a single Ubl polypeptide is attached at the site of conjugation rather than a chain as for ubiquitin. In addition, Ubls do not target the modified substrate for degradation. Rather, the modification appears to have a number of functions depending on the substrate. In some cases the modification is important for cellular localization while in others, the modified protein is stabilized. Members of both the SUMO and RUB families have been identified in plants (Vierstra and Callis, 1999).

\section{Auxin-response mutants in Arabidopsis}

By utilizing the effects of applied auxin on root elongation, a large number of auxin response mutants have been identified in Arabidopsis (Hobbie et al., 1994). Two general classes of mutants have been recovered. One class is composed of the recessive lossof-function mutations axrl, axr4, and tirl. The axrl mutants were the first to be characterized in this group (Lincoln et al., 1990). Axrl plants display a number 
of severe auxin-related growth defects including reduced apical dominance, reduced cell elongation and reduced tropic responses. The tirl (transport inhibitor response 1) mutants were initially isolated in a screen for resistance to auxin transport inhibitors, and later identified as defective in auxin response rather than auxin transport (Ruegger et al., 1998). Mature tirl plants are similar to wild-type plants in appearance. However, many auxin responses, including auxin inhibition of root growth, lateral root formation and auxin dependent hypocotyl elongation in seedlings are diminished in these mutants. The axr4 mutants also show defects in many aspects of auxin response (Hobbie and Estelle, 1995). The tirl and axr4 mutations both act synergistically with axrl suggesting that these genes act in the same or overlapping pathways (Hobbie and Estelle, 1995; Ruegger et al., 1998).

The second class of mutations confer a dominant gain-of-function. These mutants include $a x r 2$, axr3, and iaa28 (Rouse et al., 1998; Nagpal et al., 2000; Rogg et al., 2001). The related mutants shy2 and $m s g 2$ also belong to this class (Tian and Reed, 1999). In general, these mutations confer reduced auxin response and result in a variety of growth defects.

\section{The TIR1 protein, an SCF connection}

The TIR1 protein encodes a 594 amino acid protein that contains an F-box domain and 16 degenerate leucine rich repeats (LRR) (Ruegger et al., 1998). The F-box motif is located at the N-terminus, and is required for the assembly of the F-box protein onto the core of the SCF complex (cullin, Rbx 1 and Skp1) through binding to Skp1 (Patton et al., 1998). In a yeast two-hybrid screen, TIR1 interacted with two highly related genes, ASK1 (Arabidopsis Skp1like1) and ASK2 (Gray et al., 1999). Additionally, extracts from transgenic plants carrying c-myc-TIR1 co-immunoprecipitated both ASK1 and ASK2, further confirming the in vivo interactions between TIR 1 and ASK proteins (Gray et al., 1999; Gray and Estelle, 2000). The two-hybrid interaction between TIR1 and ASK1/ASK2 was completely abolished when the Fbox motif was mutated. Interestingly, this point mutation did not interfere with co-immunoprecipitation of ASK proteins with TIR1 suggesting that other regions on the protein contribute to stability of the complex. Recent experiments have linked ASK1 with auxin response. Knockout ask 1-1 mutants are male sterile and show auxin-resistant root elongation (Gray et al.,
1999; Yang et al., 1999). The Arabidopsis genome encodes at least 18 ASK proteins (Crosby, personal communication). Because the yeast two-hybrid analysis recovered only two of these, it is likely that individual ASK proteins are specialized for specific SCF complexes. There are 6 known cullin proteins in Arabidopsis. So far only AtCull is known to function in $\mathrm{SCF}^{\mathrm{TIR} 1}$ (Gray et al., 1999).

Leucine-rich repeats are found in many F-box proteins and are thought to interact with substrates, conferring specificity to the SCF complex (Patton et al., 1998). Since the tirl mutants are loss-of-function mutants, at least some TIR1 substrates must be negative regulators of auxin response. The levels of TIR1 appear to be important for auxin response, since plants that are heterozygous for tirl have an intermediate level of auxin resistance. In fact, over-expression of TIR1 confers increased auxin response (Gray et al., 1999).

\section{Auxin response and the RUB conjugation pathway}

Recessive mutations in the $A X R 1$ gene confer an array of auxin-related phenotypes, placing AXR1 in a central position in auxin response (Lincoln et al., 1990; Hobbie et al., 1994). Molecular cloning of the AXRl gene demonstrated that the protein is related to the N-terminus of ubiquitin activating (E1) enzyme (Leyser et al., 1993). AXR1-related proteins were later identified in other organisms including man and yeasts (Hochstrasser, 2000). These enzymes were shown to activate various ubiquitin-like proteins with the co-operation of a second subunit related to the C-terminus of E1. The second subunit carries the active-site cysteine.

The $A X R l$ orthologùe in budding yeast is ENR2 (E1 amino terminus-related 2). Genetic studies indicated that ENR2 is essential for modification of Cdc53 with Rub1. Although not essential for viability, this modification is required for normal function of $\mathrm{SCF}^{C D C 4}$ in the degradation of the CDK inhibitor Sic1 (Lammer et al., 1998). These results demonstrated for the first time that members of the cullin family are modified by RUB1, and that this modification is important for SCF function. In Arabidopsis, AXR1 functions together with ECR1 (E1 C-terminusrelated) to activate RUB1 and form a thiol ester linkage between RUB1 and a conserved cysteine on ECR1 in vitro (del Pozo et al., 1998). Mutation of this cysteine (C215A) completely abolishes the activity of 
the enzyme. The RCE1 (RUB1-conjugating enzyme1) gene was subsequently identified because of its similarity to yeast Ubc12. In the presence of AXR1, ECR1 and ATP, RCE1 can form a thiol ester linkage with RUB1 confirming that this protein is a RUB E2 (del Pozo and Estelle, 1999).

Based on the yeast studies, we proposed that the Arabidopsis cullin AtCul1 may be modified by RUB1. This has now been confirmed in vitro and in vivo (del Pozo and Estelle, 1999; del Pozo and Estelle, 2002). In addition, our recent studies confirm that RUB1 modification of CUL1 is dependent upon both AXR1 and ECR1 (del Pozo et al., 2002). These results are consistent with our earlier genetic studies demonstrating an interaction between the axrl and tirl mutations (Ruegger et al., 1998). It now appears very likely that the primary function of AXR1 in auxin response is through RUB1 modification of CUL1 (Figure 1).

It is noteworthy that there are at least 3 RUB/NEDD8 family proteins in Arabidopsis (RaoNaik et al., 1998). RUB1 and RUB2 differ from each other by a single amino acid, while RUB3 is about $78 \%$ identical to the other two (Rao-Naik et al., 1998). Whether RUB2 and RUB3 proteins can modify cullin proteins is currently unknown, and worth investigating. The presence of another AXR1-like protein (AXL1), another RCE1-like protein (RCE2), and four additional cullins (AtCul2-5) in the Arabidopsis proteome may suggest combinatorial regulation of additional SCF complexes employing different Fbox proteins and potential targets in a tissue-specific manner. In fact, double mutants between axrl and axll display severe auxin-related morphological defects suggesting a role for AXL1 in the same or a redundant pathway in auxin response (N. Dharmasiri and M. Estelle, unpublished results). There are also 3 other F-box proteins (LRF1, LRF2, and LRF3) with significant sequence similarity to TIR1 along the entire length of the protein encoded in the Arabidopsis genome (Dharmasiri et al., unpublished results). These proteins may also function in auxin response, a possibility that is consistent with the relatively weak phenotype of the tirl mutants.

\section{Regulation of SCF ${ }^{\mathrm{TIR} 1}$ complex}

Why is RUB1 modification of CUL1 important for function of $\mathrm{SCF}^{\mathrm{TIR} 1}$ ? At this point the answer to this question is not clear. In one study the modification appeared to be important for centrosomal localization of CUL1 (Freed et al., 1999). The RUB conjugation pathway is present in the nucleus of both plant and animal cells and it is possible that the modification helps to retain the SCF in the nucleus or target it to a specific structure within the nucleus (del Pozo et al., 1998; Yeh et al., 2000). A number of other studies indicate that the modification increases SCF activity in vitro (Morimoto et al., 2000; Podust et al., 2000; Read et al., 2000; Wu et al., 2000; Tanaka et al., 2001). At present there is no information on how this might happen. Some of the obvious possibilities have been excluded. For example, the modification does not appear to affect assembly or stability of the SCF (Yeh et al., 2000). Neither does it seem to influence the interaction between the SCF and substrates.

Recently some important clues to this puzzle have emerged from genetic studies in Arabidopsis and the yeast Schizosaccharomyces pombe (Lyapina et al., 2001; Schwechheimer et al., 2001). Two groups have shown in these species, that the COP9 signalosome (CSN) possess an activity that removes RUB/NEDD8 from cullin. The CSN is a large multisubunit complex related to the 19S lid subcomplex of the proteasome (Wei and Deng, 1999). The complex was first identified in Arabidopsis as a negative regulator of photomorphogenesis (Wei and Deng, 1999). The CSN is found in plants, animals, fission yeast, but not budding yeast. In Arabidopsis, the complete absence of the complex results in early seedling lethality. However, Schwechheimer et al. (2001) generated transgenic lines with reduced levels of the CSN5 protein, a subunit of the complex. These plants had an increased level of modified CUL1 compared to wild type suggesting that the CSN is responsible for removing RUB from the cullin. Strong support for this idea comes from the companion paper in which purified mammalian CSN was shown to remove Nedd8 from the fission yeast cullin Pcu1 in vitro. Strikingly, the CSN5 line is also resistant to auxin and has a morphology that is quite similar to that of axrl plants. Furthermore, the rate of degradation of PSIAA6, a potential target of SCF complex (see below), was reduced in the CSN5 transgenic lines. Thus an increase in RUB-modified cullin has the same effect on auxin response as a decrease in the amount of modified cullin ( $\operatorname{xxrl}$ plants). A very similar story emerges from a series of experiments involving the RBX1 protein. This subunit of the $\mathrm{SCF}$ is known to be essential for RUB-modification of the cullin (Kamura et al., 1999). In Arabidopsis plants that over-express RBX1, there is a dramatic increase in the relative amount of modified cullin (Gray 


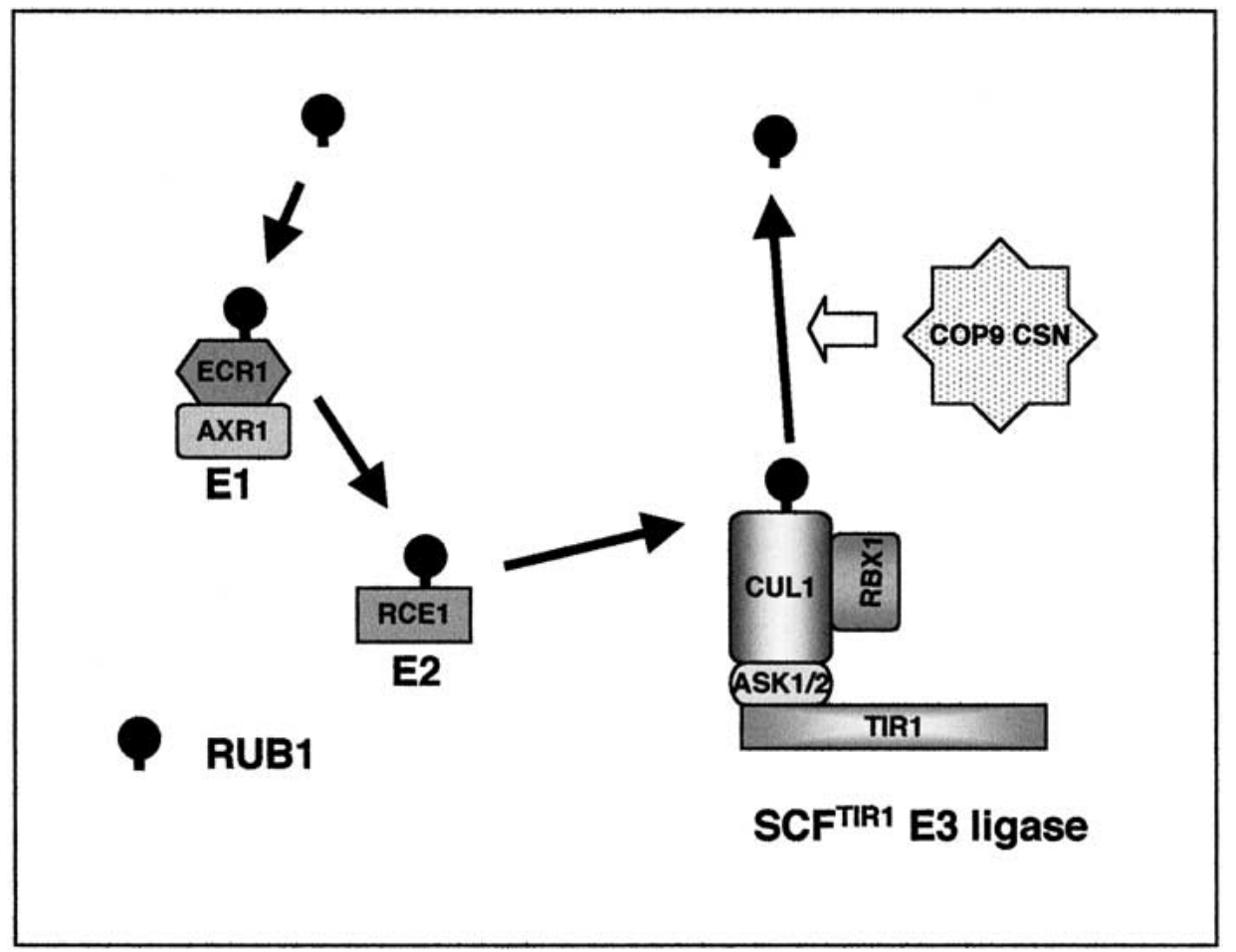

Figure 1. Model for the RUB1 conjugation / deconjugation pathway. RUB1 is activated by AXR1-ECR1 heterodimer (E1), and conjugated to CUL1 via the action of RUB1 conjugating enzyme, RCE1 (E2). The COP9 signalosome (CSN) regulates the deconjugation of RUB1 from CUL1. Activity of SCF ${ }^{\text {TIR1 }}$ E3 ligase complex appears to be regulated by RUB1 conjugation and deconjugation of CUL1 for normal auxin response.

and Estelle, unpublished). This change is associated with a reduction in auxin response and a similar suite of growth defects as is observed in the axrl and the CSN5 transgenic lines. Taken together, these results suggest that cullin modification and RUB-removal are regulated and dynamic processes. Perhaps RUB conjugation and removal are each required at specific moments in the SCF catalytic cycle.

\section{Targets of the SCF ${ }^{\text {TIR1 }}$ complex}

Considering the prominent role of SCF ${ }^{\mathrm{TIR} 1}$ in auxin response, one of the most important and interesting questions is the identity of its substrates. One group of likely candidates are the AUX/IAA proteins (Abel et al., 1994; Gray and Estelle, 2000; Worley et al., 2000). These proteins are short-lived nuclear localized proteins that have been implicated in auxin response. There are 24 Aux/IAA genes in the Arabidopsis genome, many of which are auxin regulated. The Aux/IAA proteins all have a characteristic structure with four conserved domains (I-IV). Domains III and IV are involved in homo- and hetero-dimerization within the family or with members of the ARF protein family (auxin-response factor)(Abel and Theologis, 1996; Guilfoyle et al., 1998). The ARF proteins share domains III and IV with the Aux/IAA proteins and also have a B1 DNA binding domain that is required for binding to a DNA sequence called the auxinresponse element (AuxRe) (Guilfoyle et al., 1998). ARF proteins can act as either transcriptional activators or repressors (Ulmasov et al., 1999). In at least some cases, Aux/IAA proteins inhibit ARF-mediated transcriptional regulation (Ulmasov et al., 1997). Although not yet proven, these results suggest that the Aux/IAA proteins inhibit ARF function through formation of an Aux/IAA-ARF heterodimer (Guilfoyle et al., 1998). Since the ARFs can have either positive or negative effects on transcription, this implies that the AUX/IAA proteins can act as either positive or negative regulators of auxin response.

A growing number of $A u x / I A A$ genes have been identified through genetic studies including AXR2/IAA7, AXR3/IAA17, SHY2/IAA3, and IAA28 (Morimoto et al., 2000; Read et al., 2000; Rogg 


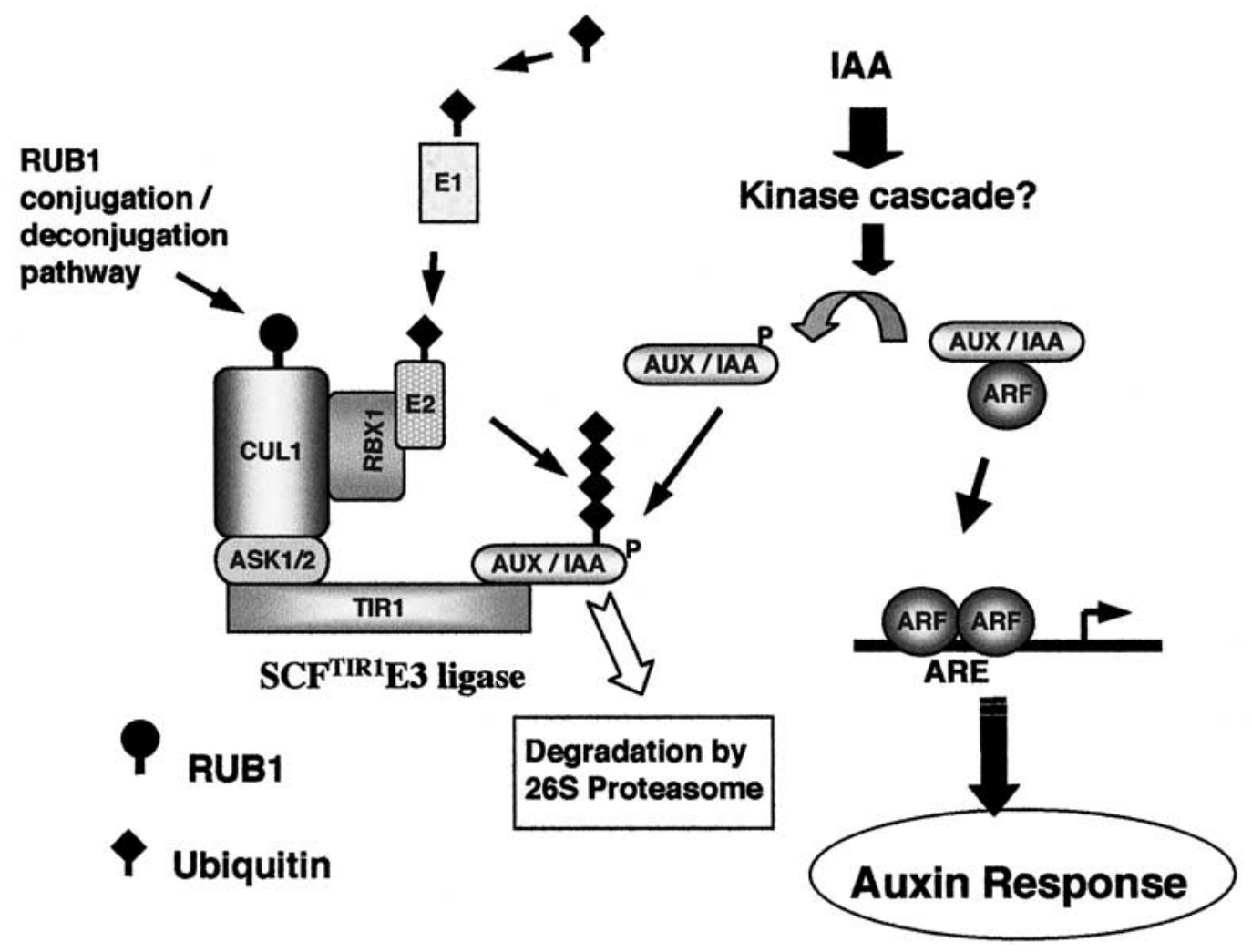

Figure 2. Model for the function of $\mathrm{SCF}^{\mathrm{TIR} 1}$ complex during auxin response. In this model we suggest that under un-induced conditions, AUX/IAA proteins remain bound to ARFs (auxin-response factors) and prevent their function. Auxin may induce the ubiquitin proteasome pathway-mediated degradation of AUX/IAA proteins, releasing ARFs. Dimerized ARFs then bind to AuxRE (auxin-response elements) in auxin-response gene promoters. It is not yet clear if an auxin-induced kinase cascade is directly involved in phosphorylation of AUX/IAA proteins or a factor associated with AUX/IAAs.

et al., 2001; Tanaka et al., 2001) (see Liscum and Reed, 2002). Gain-of-function mutations in each of these genes resulted in defects in auxin response and auxin-regulated growth processes. Molecular analysis of the mutant genes revealed that in every case, the phenotype was caused by an amino acid substitution within domain II of the protein. In fact, subsequent studies showed that domain II includes an instability determinant and the gain-of-function mutations result in stabilization of the affected protein (Worley et al., 2000).

All of these results suggest that the Aux/IAA proteins might be substrates for $\mathrm{SCF}^{\mathrm{TIR} 1}$. This has now been confirmed using genetic and biochemical ap- proaches. An AXR3-GUS fusion protein is unstable in a wild-type line and further destabilized by auxin (Gray et al., 2001). However, the fusion is more stable in both the tirl and axrl mutants suggesting that $\mathrm{SCF}^{\mathrm{TIR} 1}$ is required for normal degradation of the protein. Similar results were obtained with an AXR2-GUS fusion protein.

Most strikingly, recent studies show that both recombinant AXR2 and AXR3 interact with $\mathrm{SCF}^{\mathrm{TIR} 1}$ when added to plant extracts (Gray et al., 2001). Further, this interaction was stimulated by auxin pretreatment of the seedlings used to prepare the extract. This result suggests that the interaction between $\mathrm{SCF}^{\mathrm{TIR} 1}$ and the Aux/IAA proteins is auxin regulated. Finally, 
the domain II mutant proteins corresponding to the gain-of-function forms of AXR2 and AXR3 did not interact with the SCF indicating that stabilization of mutant protein is caused by the failure to interact with the SCF (Gray et al., 2001).

\section{Auxin regulation of Aux/IAA degradation}

A model describing our current view of $\mathrm{SCF}^{\mathrm{TIR} 1} \mathrm{reg}$ ulation of auxin response is outlined in Figure 2. The results described above largely confirm many aspects of this model. However, a major outstanding question concerns auxin regulation of the pathway. The genes in the RUB conjugation pathway (AXR1, ECR1, and $R C E 1)$ are not rapidly induced by auxin treatment and auxin does not appear to affect the level of RUB-CUL1 (del Pozo et al., 2002). Similarly, expression of TIRI is not rapidly auxin-regulated (Gray et al., 1999). Instead, all of these genes are expressed in meristems, organ primordia and other growing cells suggesting that auxin regulation of the pathway occurs by some other mechanism (Gray et al., 1999; del Pozo et al., unpublished results). As mentioned above, one possibility is through regulation of the interaction between $\mathrm{SCF}^{\mathrm{TIR} 1}$ and the Aux/IAA proteins. In most cases, SCF-substrate recognition requires phosphorylation of the substrate (Hershko and Ciechanover, 1998). Auxin may act to phosphorylate either the Aux/IAA proteins or an unknown adapter protein, thereby promoting interaction with the SCF. It is interesting to note that phytochrome A has recently been shown to phosphorylate Aux/IAA proteins in vitro (Colon-Carmona et al., 2000). At this point, the physiological significance of this activity is unclear, but it is certainly possible to imagine that PHYA acts to regulate degradation of these proteins. This would be consistent with growing evidence for a close relationship between light and hormone signaling.

\section{Conclusions and future prospects}

This review and the others in this issue demonstrate how far we have progressed in the past several years. At the same time, our recent progress has led us to a fuller understanding of the complexity of the auxin regulatory system. The recent completion of the Arabidopsis genome clearly illustrates the challenge. According to the latest annotation information there are $24 A u x / I A A$ genes, $23 A R F$ genes, and 3 genes closely related to TIR1. The other well-known auxin-regulated genes, GH3 and SAUR, are also represented by very large families in the Arabidopsis genome. There is also extraordinary complexity in the ubiquitin pathway. In addition to the $C U L$ and $A S K$ gene families, there are over 350 different F-box proteins in the proteome and similar numbers of other families of E3 ubiquitin ligases. To understand this complexity we will need to utilize all of the resources of the post-genomic era including forward and reverse genetic procedures, expression profiling, metabolic profiling and advanced proteomics. Of equal importance, an unprecedented level of cooperation and collaboration will be required for this effort. This volume was inspired by a meeting devoted to auxin biology that took place in Corsica in May, 2000. Judging by the collegial and convivial atmosphere of that meeting, the auxin community is up to this challenge.

\section{Acknowledgements}

Research in our lab is supported by grants from the NIH (43644), NSF (PGR-0077769), and the US Department of Energy (DE-FG02-98-ER20313).

\section{References}

Abel, S., Oeller, P.W. and Theologis, A. 1994. Early auxin-induced genes encode short-lived nuclear proteins. Proc. Natl. Acad. Sci. USA 91: 326-330.

Abel, S. and Theologis, A. 1996. Early genes and auxin action. Plant Physiol. 111: 9-17.

Callis, J. and Vierstra, R.D. 2000. Protein degradation in signaling. Curr. Opin. Plant Biol. 3: 381-386.

Carrano, A.C., Eytan, E., Hershko, A. and Pagano, M. 1999. SKP2 is required for ubiquitin-mediated degradation of the CDK inhibitor p27. Nature Cell Biol. 1: 193-199.

Colon-Carmona, A., Chen, D.L., Yeh, K.C. and Abel, S. 2000. Aux/IAA proteins are phosphorylated by phytochrome in vitro. Plant Physiol. 124: 1728-1738.

del Pozo, J.C. and Estelle, M. 1999. The Arabidopsis cullin AtCUL1 is modified by the ubiquitin-related protein RUB1. Proc. Natl. Acad. Sci. USA 96: 15342-15347.

del Pozo, J.C., Timpte, C., Tan, S., Callis, J. and Estelle, M. 1998. The ubiquitin-related protein RUB1 and auxin response in Arabidopsis. Science 280: 1760-1763.

del Pozo, J.C., Dharmasiri, S., Hellman, H., Walker, L., Gray, W.M. and Estelle, M., 2002. AXRI-ECRI-dependent conjugation of RUBI to the Arabidopsis Cullin AtCul1 is required for auxin response. Plant Cell (in press).

Dieterle, M., Zhou, Y.C., Schafer, E., Funk, M. and Kretsch, T. 2001. EID1, an F-box protein involved in phytochrome A-specific light signaling. Genes Dev. 15: 939-944.

Freed, E., Lacey, K.R., Huie, P., Lyapina, S.A., Deshaies, R.J., Stearns, T. and Jackson, P.K. 1999. Components of an SCF 
ubiquitin ligase localize to the centrosome and regulate the centrosome duplication cycle. Genes Dev. 13: 2242-2257.

Galan, J.M., Wiederkehr, A., Seol, J.H., Haguenauer-Tsapis, R., Deshaies, R.J., Riezman, H. and Peter, M. 2001. Skp1p and the F-box protein Rcylp form a non-SCF complex involved in recycling of the SNARE Snc1p in yeast. Mol. Cell Biol. 21: 3105-3117.

Gray, W.M. and Estelle, I. 2000. Function of the ubiquitinproteasome pathway in auxin response. Trends Biochem. Sci. 25: 133-138.

Gray, W.M., del Pozo, J.C., Walker, L., Hobbie, L., Risseeuw, E., Banks, T., Crosby, W.L., Yang, M., Ma, H. and Estelle, M. 1999. Identification of an SCF ubiquitin-ligase complex required for auxin response in Arabidopsis thaliana. Genes Dev. 13: 16781691.

Gray, W.M., Kepinski, S., Rouse, D., Leyser, O., and Estelle, M., 2001. Auxin regulates SCF ${ }^{\text {TIR1 }}$ - dependent degradation of AUX/IAA proteins. Nature 414: 271-276.

Guilfoyle, T., Hagen, G., Ulmasov, T. and Murfett, J. 1998. How does auxin turn on genes? Plant Physiol. 118: 341-347.

Hershko, A. and Ciechanover, A. 1998. The ubiquitin system. Annu. Rev. Biochem. 67: 425-479.

Hobbie, L. and Estelle, M. 1995. The axr4 auxin-resistant mutants of Arabidopsis thaliana define a gene important for root gravitropism and lateral root initiation. Plant J. 7: 211-220.

Hobbie, L., Timpte, C. and Estelle, M. 1994. Molecular genetics of auxin and cytokinin. Plant Mol. Biol. 26: 1499-1519.

Hochstrasser, M. 2000. Evolution and function of ubiquitin-like protein-conjugation systems. Nature Cell Biol. 2: E153-E157.

Kamura, T., Conrad, M.N., Yan, Q., Conaway, R.C. and Conaway, J.W. 1999. The Rbx1 subunit of SCF and VHL E3 ubiquitin ligase activates Rub1 modification of cullins Cdc53 and Cul2. Genes Dev. 13: 2928-2933.

Kipreos, E.T. and Pagano, M. 2000. The F-box protein family. Genome Biol. 1 (2000).

Lammer, D., Mathias, N., Laplaza, J.M., Jiang, W., Liu, Y., Callis, J., Goebl, M. and Estelle, M. 1998. Modification of yeast Cdc53p by the ubiquitin-related protein rub1p affects function of the SCFCdc4 complex. Genes Dev. 12: 914-926.

Leyser, H.M., Lincoln, C.A., Timpte, C., Lammer, D., Turner, J. and Estelle, M. 1993. Arabidopsis auxin-resistance gene AXR1 encodes a protein related to ubiquitin-activating enzyme E1. Nature 364: 161-164.

Lincoln, C., Britton, J.H. and Estelle, M. 1990. Growth and development of the axr1 mutants of Arabidopsis. Plant Cell 2: 1071-1080.

Liscum, E. and Reed, J.W. 2002. Genetics of Aux/IAA and ARF action in plant growth and development. Plant Mol. Biol. 49: $387-400$.

Lyapina, S., Cope, G., Shevchenko, A., Serino, G., Tsuge, T., Zhou, C., Wolf, D.A., Wei, N. and Deshaies, R.J. 2001. Promotion of NEDD8-CUL1 conjugate cleavage by COP9 signalosome. Science 292: 1382-1385.

Morimoto, M., Nishida, T., Honda, R. and Yasuda, H. 2000. Modification of cullin-1 by ubiquitin-like protein Nedd8 enhances the activity of SCF(skp2) toward p27(kip1). Biochem. Biophys. Res. Comm. 270: 1093-1096.

Nagpal, P., Walker, L.M., Young, J.C., Sonawala, A., Timpte, C., Estelle, M. and Reed, J.W. 2000. AXR2 encodes a member of the Aux/IAA protein family [In Process Citation]. Plant Physiol. 123: 563-574.

Nelson, D.C., Lasswell, J., Rogg, L.E., Cohen, M.A. and Bartel, B. 2000. FKF1, a clock-controlled gene that regulates the transition to flowering in Arabidopsis. Cell 101: 331-340.
Patton, E.E., Willems, A.R. and Tyers, M. 1998. Combinatorial control in ubiquitin-dependent proteolysis: don't Skp the F-box hypothesis. Trends Genet. 14: 236-243.

Podust, V.N., Brownell, J.E., Gladysheva, T.B., Luo, R.S., Wang, C., Coggins, M.B., Pierce, J.W., Lightcap, E.S. and Chau, V. 2000. A Nedd8 conjugation pathway is essential for proteolytic targeting of p27Kip1 by ubiquitination. Proc. Natl. Acad. Sci. USA 97: 4579-4584.

Rao-Naik, C., delaCruz, W., Laplaza, J.M., Tan, S., Callis, J. and Fisher, A.J. 1998. The rub family of ubiquitin-like proteins. Crystal structure of Arabidopsis rub1 and expression of multiple rubs in Arabidopsis. J. Biol. Chem. 273: 34976-34982.

Read, M.A., Brownell, J.E., Gladysheva, T.B., Hottelet, M., Parent, L.A., Coggins, M.B., Pierce, J.W., Podust, V.N., Luo, R.S., Chau, V. and Palombella, V.J. 2000. Nedd8 modification of cul-1 activates $\operatorname{SCF}(\beta(\operatorname{TrCP}))$-dependent ubiquitination of $\mathrm{I} \kappa \mathrm{B} \alpha$. Mol. Cell Biol. 20: 2326-2333.

Rogg, L.E., Lasswell, J. and Bartel, B. 2001. A gain-of-function mutation in iaa28 suppresses lateral root development. Plant Cell 13: 465-480.

Rouse, D., Mackay, P., Stirnberg, P., Estelle, M. and Leyser, O. 1998. Changes in auxin response from mutations in an AUX/IAA gene. Science 279: 1371-1373.

Ruegger, M., Dewey, E., Gray, W.M., Hobbie, L., Turner, J. and Estelle, M. 1998. The TIR1 protein of Arabidopsis functions in auxin response and is related to human SKP2 and yeast grr1p. Genes Dev. 12: 198-207.

Samach, A., Klenz, J.E., Kohalmi, S.E., Risseeuw, E., Haughn, G.W. and Crosby, W.L. 1999. The UNUSUAL FLORAL ORGANS gene of Arabidopsis thaliana is an F-box protein required for normal patterning and growth in the floral meristem. Plant $\mathrm{J}$. 20: 433-445.

Schwechheimer, C., Serino, G., Callis, J., Crosby, W.L., Lyapina, S., Deshaies, R.J., Gray, W.M., Estelle, M. and Deng, X.W. 2001. Interactions of the COP9 signalosome with the E3 ubiquitin ligase SCFTIR1 in mediating auxin response. Science 292: 1379-1382.

Somers, D.E., Schultz, T.F., Milnamow, M. and Kay, S.A. 2000. ZEITLUPE encodes a novel clock-associated PAS protein from Arabidopsis. Cell 101: 319-329.

Tanaka, K., Kawakami, T., Tateishi, K., Yashiroda, H. and Chiba, T. 2001. Control of $\mathrm{I} \kappa \mathrm{B} \alpha$ proteolysis by the ubiquitin-proteasome pathway. Biochimie 83: 351-356.

Tian, Q. and Reed, J.W. 1999. Control of auxin-regulated root development by the Arabidopsis thaliana SHY2/IAA3 gene. Development 126: 711-721.

Tyers, M. and Willems, A.R. 1999. One ring to rule a superfamily of E3 ubiquitin ligases [comment]. Science 284: 601, 603, 604.

Ulmasov, T., Murfett, J., Hagen, G. and Guilfoyle, T.J. 1997. Aux/IAA proteins repress expression of reporter genes containing natural and highly active synthetic auxin response elements. Plant Cell 9: 1963-1971.

Ulmasov, T., Hagen, G. and Guilfoyle, T.J. 1999. Activation and repression of transcription by auxin-response factors. Proc. Natl. Acad. Sci. USA 96: 5844-5849.

Vierstra, R.D. and Callis, J. 1999. Polypeptide tags, ubiquitous modifiers for plant protein regulation. Plant Mol. Biol. 41: 435-442.

Wei, N. and Deng, X.W. 1999. Making sense of the COP9 signalosome. A regulatory protein complex conserved from Arabidopsis to human. Trends Genet. 15: 98-103.

Worley, C.K., Zenser, N., Ramos, J., Rouse, D., Leyser, O., Theologis, A. and Callis, J. 2000. Degradation of Aux/IAA proteins is essential for normal auxin signalling. Plant J. 21: 553-562. 
Wu, K., Chen, A. and Pan, Z.Q. 2000. Conjugation of Nedd8 to CUL1 enhances the ability of the ROC1-CUL1 complex to promote ubiquitin polymerization. J. Biol. Chem. 275: $32317-$ 32324.

Xie, D.X., Feys, B.F., James, S., Nieto-Rostro, M. and Turner, J.G. 1998. COI1: an Arabidopsis gene required for jasmonateregulated defense and fertility. Science 280: 1091-1094.
Yang, M., Hu, Y., Lodhi, M., McCombie, W.R. and Ma, H. 1999. The Arabidopsis SKP1-LIKE1 gene is essential for male meiosis and may control homologue separation. Proc. Natl. Acad. Sci. USA 96: 11416-11421.

Yeh, E.T., Gong, L. and Kamitani, T. 2000. Ubiquitin-like proteins: new wines in new bottles. Gene 248: 1-14. 